\title{
A comparison of finite elements for nonlinear beams: the absolute nodal coordinate and geometrically exact formulations
}

\author{
Ignacio Romero
}

\begin{abstract}
Two of the most popular finite element formulations for solving nonlinear beams are the absolute nodal coordinate and the geometrically exact approaches. Both can be applied to problems with very large deformations and strains, but they differ substantially at the continuous and the discrete levels. In addition, implementation and run-time computational costs also vary significantly. In the current work, we summarize the main features of the two formulations, highlighting their differences and similarities, and perform numerical benchmarks to assess their accuracy and robustness. The article concludes with recommendations for the choice of one formulation over the other.
\end{abstract}

Keywords Nonlinear beam · Finite element · Flexible multibody · Absolute nodal coordinate $\cdot$ Geometrically exact

\section{Introduction}

Beams are among the most commonly used members in solid structural mechanics and are becoming increasingly popular in (flexible) multibody analyses. The classical EulerBernoulli and Timoshenko theories have been used in linear structural analysis for decades now, and many commercial codes employ them. In geometrically nonlinear problems, matters complicate and there is still no consensus about which beam theory should then be used and, especially, which numerical approximation needs to be chosen

Fully nonlinear beam theories have been proposed, for example, The last two publications formulate, respectively, a two dimensional beam model and its three dimensional extension which allows large strains and rotations. Despite their generality, they admit a fairly compact and simple formulation. Beam models of this type have been coined "geometrically exact" because they account without approximation for the total deformation and strains. 
The simplicity of the equations of geometrically exact beams have motivated the development of many numerical implementations in the finite element literature and related fields. See among many others,

The large number of different numerical implementations of geometrically exact beams hints at the intrinsic difficulty of the numerical discretization of the continuous equations. This issue is directly related with the presence of rotational degrees of freedom in the theory, and its discretization in the finite element models

The nonlinear character of the rotational degrees of freedom make the formulation and implementation of geometrically exact finite elements complex

Also, it requires important modifications in the global structure of standard finite element, structural, or multibody codes. To sidestep all these problems, other beam formulations have been proposed in recent years. Among them, we will discuss the so-called absolute nodal coordinate formulation of beams as proposed in

These articles advocate the use of special interpolation functions that employed in the context of a standard solid finite element are rich enough so as to faithfully represent the kinematics of a beam-like solid. The resulting method is fairly simple, but has its own drawbacks as discussed,

The geometrically exact formulations have become the dominant approach in the finite element community. In the context of flexible multibody dynamics, both types of beam approximations seem to have received similar attention. A recent comparison of both methodologies, restricted to the context of eigenvalue analysis and the effect of centrifugal forces

The present work extends this comparison and considers a wider range of aspects, trying to assess the relative strengths and weaknesses of each formulation. The goal of this article is precisely to analyze both methodologies so as to provide guidelines for the choice and use of either one. The comparison spans implementation details, robustness, computational cost, numerical issues, interface with existing codes and material models, and accuracy.

An outline of the rest of the paper is as follows. In Sects. 2 and 3, the absolute nodal coordinate and geometrically exact beam formulations are reviewed. Even though some of the implementation details are left unspecified (with references provided), these two sections provide enough information to appreciate the complexity of each formulation and the fundamental structure of the resulting equations. Next, in Sect. 4, the two formulations are compared qualitatively under a wide range of criteria. The comparison makes frequent references to the details in Sects. 2 and 3, as well as other works in the literature. To study the accuracy of the two formulations, Sect. 5 shows several numerical examples, and the results obtained with both methods are compared. Section 6 concludes the article summarizing the main findings and providing some guidelines for the choice of a particular beam formulation, based on the obtained results and the features identified in the comparison.

\section{Summary of the absolute nodal coordinates formulation}

The first beam model we review is the Absolute Nodal Coordinates (ANC) formulation,

As we shall describe next, the key point of this formulation is the choice of interpolation functions for the beam geometry and deformation. By selecting a richer interpolation space in the direction of the beam axis than in the other two spatial directions, the kinematics of the model can faithfully approximate the motions of slender bodies.

To describe the formulation, consider a mesh of the beam consisting of two-noded finite elements. A global coordinate system is selected for $\mathbb{R}^{3}$ with coordinates denoted $(X, Y, Z)$ 
Fig. 1 ANC beam

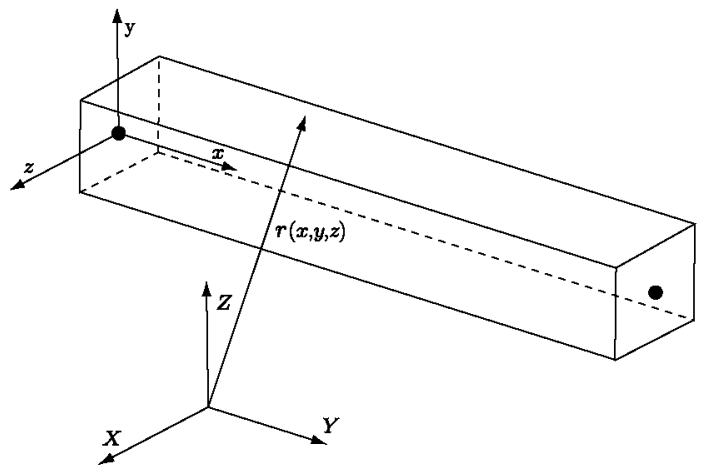

and basis $\{\mathbf{i}, \mathbf{j}, \mathbf{k}\}$. The positions of the nodes in the undeformed configuration are denoted $\mathbf{X}_{1}, \mathbf{X}_{2}, \ldots, \mathbf{X}_{n_{\text {node }}}$. In each element, a local reference system is defined with coordinates $(x, y, z)$ in such a way that the variable $x$ corresponds to the axial direction and can take values $0 \leq x \leq l_{e}, l_{e}$ being the length of element $e$. See Fig. 1 for an illustration.

The most important assumption of the ANC formulation is that a point of the beam belonging to element $e$ with local coordinates $(x, y, z)$ takes, at time $t$, the following position $\varphi$ in space:

$$
\varphi(x, y, z, t)=\mathbf{S}_{e}(x, y, z)\left\{\begin{array}{l}
\mathbf{a}_{e}(t) \\
\mathbf{b}_{e}(t) \\
\mathbf{c}_{e}(t)
\end{array}\right\} .
$$

The matrix $\mathbf{S}_{e}$ contains the element interpolation functions and it is of the form:

$$
\mathbf{S}_{e}(x, y, z)=\left[\begin{array}{ccc}
\mathbf{J}_{N}(x, y, z) & \mathbf{0}_{N} & \mathbf{0}_{N} \\
\mathbf{0}_{N} & \mathbf{J}_{N}(x, y, z) & \mathbf{0}_{N} \\
\mathbf{0}_{N} & \mathbf{0}_{N} & \mathbf{J}_{N}(x, y, z)
\end{array}\right],
$$

where $\mathbf{0}_{N}$ is the $N$-dimensional zero row vector and with the interpolation row vector

$$
\mathbf{J}_{N}(x, y, z)=\left\langle 1, x, y, z, x y, x z, x^{2}, x^{3}, \ldots, x^{N-5}\right\rangle .
$$

The constant $N$ determines the degree of polynomial interpolation in the $x$ direction and must be greater or equal to 7 .

we assume without loss of generality that $N=8$. In view of (1), the number degrees of freedom per element is $3 \times N$.

The vector $\left\langle\mathbf{a}_{e}(t), \mathbf{b}_{e}(t), \mathbf{c}_{e}(t)\right\rangle^{T}$ contains the coefficients of the element interpolation functions. Let $\mathbf{H}_{e}^{\alpha}$, for $\alpha=1,2$, be the following set of 12 variables associated with the local node $\alpha$ belonging to element $e$ :

$$
\mathbf{H}_{e}^{\alpha}(t)=\left\langle\boldsymbol{\varphi}\left(\mathbf{X}_{e}^{\alpha}, t\right), \frac{\partial \boldsymbol{\varphi}\left(\mathbf{X}_{e}^{\alpha}, t\right)}{\partial x}, \frac{\partial \boldsymbol{\varphi}\left(\mathbf{X}_{e}^{\alpha}, t\right)}{\partial y}, \frac{\partial \boldsymbol{\varphi}\left(\mathbf{X}_{e}^{\alpha}, t\right)}{\partial z}\right\rangle^{T}, \quad \alpha=1,2 .
$$

Note that the three last vectors of $\mathbf{H}_{e}^{\alpha}$ are the three tangent vectors to the local coordinate curves $x, y$, and $z$.

It can be verified that for the specific formulation considered herein $(N=8)$, the deformation $\varphi$ in element $e$ can be expressed not only as in (1) but also as:

$$
\boldsymbol{\varphi}(x, y, z, t)=\mathbf{D}_{e}(x, y, z)\left\{\begin{array}{l}
\mathbf{H}_{e}^{1}(t) \\
\mathbf{H}_{e}^{2}(t)
\end{array}\right\},
$$


for a new interpolation matrix $\mathbf{D}_{e}$ related to $\mathbf{S}_{e}$. Although completely equivalent to expression (1), this new interpolation has the advantage that the set of variables $\left\langle\mathbf{H}_{e}^{1}, \mathbf{H}_{e}^{2}\right\rangle^{T}$ has a more clear geometrical meaning that the polynomial coefficients $\left\langle\mathbf{a}_{e}, \mathbf{b}_{e}, \mathbf{c}_{e}\right\rangle^{T}$. More importantly, the former can be related to the global nodal degrees of freedom

$$
\mathbf{H}_{a}(t)=\left\langle\boldsymbol{\varphi}\left(\mathbf{X}_{e}^{\alpha}, t\right), \frac{\partial \varphi\left(\mathbf{X}_{e}^{\alpha}, t\right)}{\partial X}, \frac{\partial \varphi\left(\mathbf{X}_{e}^{\alpha}, t\right)}{\partial Y}, \frac{\partial \varphi\left(\mathbf{X}_{e}^{\alpha}, t\right)}{\partial Z}\right)^{T}, \quad a=1,2, \ldots, n_{\text {node }},
$$

by means of a transformation matrix $\mathbf{T}_{e}^{\alpha}$ that depends only on the undeformed configuration of the beam

$$
\mathbf{H}_{e}^{\alpha}(t)=\mathbf{T}_{e}^{\alpha}\left(\mathbf{X}_{e}^{1}, \mathbf{X}_{e}^{2}\right) \mathbf{H}_{a}(t) .
$$

In the previous two equations, the node with global label $a$ coincides with the node with local label $\alpha$ in element $e$. In the case in which the undeformed beam consists of straight elements, the matrices $\mathbf{T}_{e}^{\alpha}$ are of the form

$$
\mathbf{T}_{e}^{\alpha}\left(\mathbf{X}_{e}^{1}, \mathbf{X}_{e}^{2}\right)=\left[\begin{array}{cccc}
\mathbf{I} & \mathbf{0} & \mathbf{0} & \mathbf{0} \\
\mathbf{0} & \left(\mathbf{Q}_{e}^{\alpha}\right)_{11} \mathbf{I} & \left(\mathbf{Q}_{e}^{\alpha}\right)_{21} \mathbf{I} & \left(\mathbf{Q}_{e}^{\alpha}\right)_{31} \mathbf{I} \\
\mathbf{0} & \left(\mathbf{Q}_{e}^{\alpha}\right)_{12} \mathbf{I} & \left(\mathbf{Q}_{e}^{\alpha}\right)_{22} \mathbf{I} & \left(\mathbf{Q}_{e}^{\alpha}\right)_{32} \mathbf{I} \\
\mathbf{0} & \left(\mathbf{Q}_{e}^{\alpha}\right)_{13} \mathbf{I} & \left(\mathbf{Q}_{e}^{\alpha}\right)_{23} \mathbf{I} & \left(\mathbf{Q}_{e}^{\alpha}\right)_{33} \mathbf{I}
\end{array}\right],
$$

where $\mathbf{Q}_{e}^{\alpha}$ is the rotation matrix that transforms the local element axes to the global axes and $\mathbf{I}$ the $3 \times 3$ identity matrix.

$\mathrm{Up}$ to this point, we have described the basic kinematic assumptions of the ANC formulation. Based on them, a beam structural model could be developed, such as in [35, 37, 44]. It is more common, however, to proceed along the lines originally presented in [45], which essentially amount to using the former kinematic framework within the context of a standard finite strain, finite element formulation. This choice makes the rest of the method fairly straightforward, and details about such finite elements can be found in standard references such as

For completeness, we summarize the main steps next.

From the expression (5) of the deformation, we can compute the deformation gradient $\mathbf{F}=\frac{\partial \varphi}{\partial \mathbf{X}}$, and nonlinear strain measures such as the right Cauchy-Green tensor $\mathbf{C}$ and the Green-Lagrange tensor $\mathbf{E}$ :

$$
\mathbf{C}=\mathbf{F}^{T} \mathbf{F}, \quad \mathbf{E}=\frac{1}{2}(\mathbf{C}-\mathbf{I}) .
$$

From either of these strain measures, or any other properly defined one, the Cauchy stress tensor $\sigma$ can be obtained

$$
\boldsymbol{\sigma}=\frac{1}{J} \mathbf{F} \hat{\mathbf{S}}(\mathbf{E}) \mathbf{F}^{T}, \quad J=\operatorname{det}(\mathbf{F})
$$

In the previous equation, $\hat{\mathbf{S}}$ denotes any elastic constitutive equation. In fact, the model defined hereto is not restricted to elasticity and it can be employed, only by using the appropriate constitutive equation to study, for instance, plastic and viscoplastic beams.

Finally, the dynamic equilibrium of the beam is weakly imposed by the dynamic principle of virtual work. To define it completely, let us consider a beam of total length $L$, with (possibly variable) cross section $\mathcal{S}$, of material with density $\rho$. If the beam is under body forces $\mathbf{B}$ and surface tractions $\mathbf{T}$, the dynamic equilibrium of linear momentum can be stated 
as:

$$
\begin{array}{r}
\int_{0}^{L} \int_{\mathcal{S}} \boldsymbol{\sigma}: \operatorname{grad} \delta \boldsymbol{\varphi} \mathrm{d} S \mathrm{~d} x+\int_{0}^{L} \int_{\mathcal{S}} \ddot{\boldsymbol{\varphi}} \cdot \delta \boldsymbol{\varphi} \mathrm{d} S \mathrm{~d} x \\
=\int_{0}^{L} \int_{\mathcal{S}} \mathbf{B} \cdot \delta \boldsymbol{\varphi} \mathrm{d} S \mathrm{~d} x+\int_{0}^{L} \int_{\partial \mathcal{S}} \mathbf{T} \cdot \delta \boldsymbol{\varphi} \mathrm{d} \Gamma \mathrm{d} x,
\end{array}
$$

for all admissible variation $\delta \varphi$ of the deformation. In the previous equation, the symbol 'grad' denotes the gradient with respect to the deformed configuration of the beam.

Several important consequences stem from this variational form. The first one, as already mentioned, is that it is identical to that employed for standard finite strain solid elements. The second one is that by writing the contribution to the second integral of element $e$ as:

$$
\begin{aligned}
\int_{0}^{l_{e}} \int_{\mathcal{S}} \ddot{\boldsymbol{\varphi}} \cdot \delta \varphi \mathrm{d} S \mathrm{~d} x & =\left\{\begin{array}{l}
\delta \mathbf{H}_{e}^{1} \\
\delta \mathbf{H}_{e}^{2}
\end{array}\right\} \cdot \int_{0}^{l_{e}} \int_{\mathcal{S}} \mathbf{D}_{e}^{T} \mathbf{D}_{e} \mathrm{~d} S \mathrm{~d} x\left\{\begin{array}{c}
\ddot{\mathbf{H}}_{e}^{1} \\
\ddot{\mathbf{H}}_{e}^{2}
\end{array}\right\} \\
& =\left\{\begin{array}{l}
\delta \mathbf{H}_{e}^{1} \\
\delta \mathbf{H}_{e}^{2}
\end{array}\right\} \cdot \mathbf{M}_{e}\left\{\begin{array}{l}
\ddot{\mathbf{H}}_{e}^{1} \\
\ddot{\mathbf{H}}_{e}^{2}
\end{array}\right\},
\end{aligned}
$$

we identify the term of the integral as the element mass matrix $\mathbf{M}_{\ell}$, which is constant in time and independent of the deformation.

The final observation is that the mechanical meaning of the terms appearing in the calculation of the external virtual work is not clear. To see this it suffices, for instance, to examine the contribution of the body forces in one element:

$$
\int_{0}^{l_{e}} \int_{S} \mathbf{B} \cdot \delta \varphi \mathrm{d} S \mathrm{~d} x=\left\{\begin{array}{l}
\delta \mathbf{H}_{e}^{1} \\
\delta \mathbf{H}_{e}^{2}
\end{array}\right\} \cdot \int_{0}^{l_{e}} \int_{S} \mathbf{D}_{e}^{T} \mathbf{B} \mathrm{d} S \mathrm{~d} x=\left\{\begin{array}{l}
\delta \mathbf{H}_{e}^{1} \\
\delta \mathbf{H}_{e}^{2}
\end{array}\right\} \cdot \mathbf{F}_{e} .
$$

The element external force vector $\mathbf{F}_{e}$ is, for the case considered $(N=8)$, a vector of dimension 24 . The physical interpretation of some of its entries is not easy to grasp.

\section{Summary of the formulation of geometrically exact beam models}

As explained in the Introduction, among the several Geometrically Exact (GE) beam theories,

This is probably the most popular theory of this kind and we refer to the cited article for complete details.

A beam is defined as a slender body that can be described mathematically with a curve of centroids and a section $A$ attached to each point of this curve. Let $S$ denote the arc-length of the centroid curve and $\{\mathbf{i}, \mathbf{j}, \mathbf{k}\}_{i=1}^{3}$ the Cartesian basis of $\mathbb{R}^{3}$. Then the position vector of any point of the beam at time $t$ is given by

$$
\varphi\left(S, \xi^{1}, \xi^{2}, t\right)=\mathbf{r}(S, t)+\xi\left(S, \xi^{1}, \xi^{2}, t\right) \quad \text { with } \xi=\sum_{\alpha=1}^{2} \xi^{\alpha} \mathbf{d}_{\alpha}(S, t)
$$

The vector $\mathbf{r}$ is the position of a point on the centroid curve and $\boldsymbol{\xi}$ is a vector in the cross section. The two directors $\mathbf{d}_{\alpha}$ span the cross section of the beam at the point $\mathbf{r}$, and $\xi^{\alpha}$ are the section coordinates. In the beam model considered, we always take $\mathbf{d}_{1}$ and $\mathbf{d}_{2}$ to be orthonormal, and we define the rotation tensor $\boldsymbol{\Lambda}=\left[\mathbf{d}_{1}, \mathbf{d}_{2}, \mathbf{d}_{3}\right]$ with $\mathbf{d}_{3}=\mathbf{d}_{1} \times \mathbf{d}_{2}$. See 
Fig. 2 Geometry of a geometrically exact beam

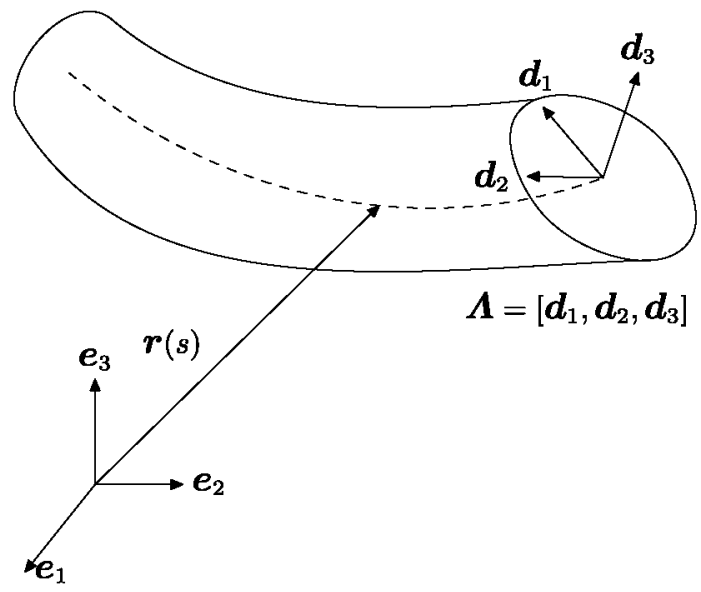

Fig. 2. Hereafter, a superscript $(\cdot)^{o}$ will denote a quantity on the reference configuration. To simplify the theory, we choose $\mathbf{d}_{3}^{o}=\mathbf{r}_{, s}^{o}$.

Two frame invariant convected strain measures are defined as

$$
\begin{aligned}
\boldsymbol{\Gamma} & =\boldsymbol{\Lambda}^{T} \mathbf{r}_{, s}-\mathbf{k}, \\
\hat{\boldsymbol{\Omega}} & =\boldsymbol{\Lambda}^{T} \boldsymbol{\Lambda}_{, s}-\boldsymbol{\Lambda}^{o, T} \boldsymbol{\Lambda}_{, s}^{o},
\end{aligned}
$$

which completely characterize the deformation of the rod. In the preceding equation, and for the rest of this article, we denote by $\mathbf{a}$ the skew symmetric tensor with axial vector $\mathbf{a} \in \mathbb{R}^{3}$. Work conjugate with the strain measures (14), we define stress resultants $\mathbf{N}, \mathbf{M}$ which for a hyperelastic material are obtained from a stored energy function $W(\boldsymbol{\Gamma}, \boldsymbol{\Omega})$ through the relations

$$
\mathbf{N}=\frac{\partial W}{\partial \boldsymbol{\Gamma}}, \quad \mathbf{M}=\frac{\partial W}{\partial \boldsymbol{\Omega}} .
$$

The first resultant includes the shear forces in the directions of the two directors and the axial force. The second resultant holds the two bending moments with respect to the directors as well as the torsion on the section. The simplest section constitutive law, and the one used in all our examples is given by:

$$
\mathbf{N}=\left[\begin{array}{ccc}
G \bar{A} & 0 & 0 \\
0 & G \bar{A} & 0 \\
0 & 0 & E A
\end{array}\right] \boldsymbol{\Gamma}, \quad \mathbf{M}=\left[\begin{array}{ccc}
E I_{1} & 0 & 0 \\
0 & E I_{2} & 0 \\
0 & 0 & G J
\end{array}\right] \mathbf{\Omega},
$$

where $E, G$ are, respectively, Young's modulus and the shear modulus; $I_{1}, I_{2}, J, \bar{A}$ are the two principal moments of inertia, the torsional stiffness, and the reduced section, respectively.

Spatial stress resultants are obtained by pushing forward with the rotation $\boldsymbol{\Lambda}$ the two convected resultants:

$$
\mathbf{n}=\mathbf{\Lambda} \mathbf{N}, \quad \mathbf{m}=\mathbf{\Lambda} \mathbf{M}
$$

The time derivative of the rotation of a section can be expressed alternatively as

$$
\dot{\boldsymbol{\Lambda}}=\hat{\boldsymbol{\omega}} \boldsymbol{\Lambda}=\boldsymbol{\Lambda} \hat{\mathbf{\Omega}}
$$


The vectors $\boldsymbol{\Omega}, \boldsymbol{\omega}$ are, respectively, the body and spatial angular velocities of the corresponding section. Consider finally $\tilde{\mathbf{n}}, \tilde{\mathbf{m}}$ to be the distributed force and moment per unit length. With these definitions, the strong form of the equilibrium equations of a beam with density $\rho$ can be written as:

$$
\begin{aligned}
\mathbf{n}_{, s}+\tilde{\mathbf{n}} & =A_{\rho} \ddot{\varphi}, \\
\mathbf{m}_{, s}+\mathbf{r}_{, s} \times \mathbf{n}+\tilde{\mathbf{m}} & =\frac{\mathrm{d}}{\mathrm{d} t}\left(\mathbf{i}_{\rho} \boldsymbol{\omega}\right) .
\end{aligned}
$$

The section area and inertia $A_{\rho}, \mathbf{i}_{\rho}$ are defined as:

$$
A_{\rho}=\rho A, \quad \mathbf{i}_{\rho}=\int_{A} \rho\left(|\xi|^{2} 1+\xi \otimes \xi\right) \mathrm{d} A .
$$

To complete the definition of the whole dynamic problem, the previous equations must be supplemented with boundary conditions

$$
\begin{aligned}
& \mathbf{r}=\overline{\mathbf{r}}, \quad \boldsymbol{\Lambda}=\overline{\boldsymbol{\Lambda}} \quad \text { on } \Gamma_{g}, \\
& \mathbf{n}=\overline{\mathbf{n}}, \quad \mathbf{m}=\overline{\mathbf{m}} \quad \text { on } \Gamma_{h}, \\
& \Gamma_{g} \cap \Gamma_{h}=\varnothing, \quad \Gamma_{g} \cup \Gamma_{h}=\{0, L\},
\end{aligned}
$$

and initial conditions

$$
\begin{array}{ll}
\mathbf{r}(S, 0)=\mathbf{r}_{o}(S), & \dot{\mathbf{r}}(S, 0)=\mathbf{v}_{o}(S), \\
\mathbf{\Lambda}(S, 0)=\boldsymbol{\Lambda}_{o}(S), & \boldsymbol{\Omega}(S, 0)=\boldsymbol{\Omega}_{o}(S) .
\end{array}
$$

The variational formulation of the dynamical equilibrium states that the solution to the beam problem with boundary data (20) and initial data (21) is the motion $(\mathbf{r}(S, t), \boldsymbol{\Lambda}(S, t))$ that verifies

$$
\begin{gathered}
\int_{0}^{L}\left(\mathbf{N} \cdot \delta \boldsymbol{\Gamma}+\mathbf{M} \cdot \delta \boldsymbol{\theta}+A_{\rho} \ddot{\mathbf{r}} \cdot \delta \mathbf{r}+\frac{\mathrm{d}}{\mathrm{d} t}\left(\mathbf{i}_{\rho} \boldsymbol{\omega}\right) \cdot \delta \boldsymbol{\theta}\right) \mathrm{d} S \\
=\int_{0}^{L}(\tilde{\mathbf{n}} \cdot \delta \mathbf{r}+\tilde{\mathbf{m}} \cdot \delta \boldsymbol{\theta}) \mathrm{d} S+[\overline{\mathbf{n}} \cdot \delta \mathbf{r}+\overline{\mathbf{m}} \cdot \delta \boldsymbol{\theta}]_{\Gamma_{h}},
\end{gathered}
$$

for all admissible variations $(\delta \mathbf{r}, \delta \boldsymbol{\theta})$.

The finite element formulations of geometrically exact rods are based on Galerkin type approximations of (22). The deformation of the centroid curve is interpolated from the nodal positions using standard Lagrangian shape functions:

$$
\mathbf{r}(S, t) \approx \mathbf{r}^{h}(S, t)=\sum_{a=1}^{n_{\text {node }}} N^{a}(S) \mathbf{r}_{a}(t) .
$$

In contrast, the section orientation $\boldsymbol{\Lambda}(S, t)$ is a function on the special orthogonal group, a nonlinear manifold that does not admit linear interpolation Several solutions have been proposed in the literature, each of them resulting in a different finite element formulation, with different properties. For concreteness, we briefly describe next the implementation described in the original article To circumvent the interpolation of the rotation field, the authors propose to construct an initial rotation field on the beam, and successively update it 
by interpolating the incremental rotation vector $\boldsymbol{\theta}$. This vector belongs to $\mathbb{R}^{3}$, and it can be interpolated in a similar fashion as the centroid curve:

$$
\boldsymbol{\theta}(S, t) \approx \boldsymbol{\theta}^{h}(S, t)=\sum_{a=1}^{n_{\text {node }}} N^{a}(S) \boldsymbol{\theta}_{a}(t)
$$

Once the incremental rotation vector is interpolated to every integration point on the beam, the rotation is incremented with the exponential map:

$$
\boldsymbol{\Lambda}_{n+1}\left(S_{i}\right)=\exp \left[\theta\left(S_{i}\right)\right] \boldsymbol{\Lambda}_{n}\left(S_{i}\right)
$$

where $S_{i}$ is the coordinate of the integration point.

The interpolation of the rotation field is not the only difficulty involved in the formulation of geometrically exact beams. Once the nodal incremental rotations have been computed, they must be employed to compute the nodal values of the angular velocity and acceleration. Such updates are far from trivial. See, for example, [11, 42]. In general, the relation between the dynamic variables is nonlinear and interpolation formulas, such as the ones commonly employed in linear multistep methods need to be modified. As with the spatial interpolation, several valid options are available, each of them conferring different properties to the ensuing methods. We refer to the previous references for examples and discussion of the effects of such choice.

\section{Qualitative comparison}

After briefly describing the ANC and the GE formulations, we proceed in this section and the next one to discuss the relative advantages and disadvantages of each methodology. In the current section, we focus on qualitative aspects that can be of relevance for both the developer as well as the user of nonlinear beams, leaving quantitative considerations to Sect. 5.

We base our comments on observations made on the formulations as described in Sects. 2 and 3, providing when possible reference to the literature where more details can be obtained. The choice of topics discussed is obviously biased by our experience but tries to cover the most important aspects.

\subsection{Implementation cost}

The first issue to be weighted when considering whether to incorporate one finite element formulation or another into an existing code is the cost that will be presumably involved in its implementation.

It can be deduced from Sect. 2 that the implementation of ANC beams is quite straightforward. If the code has already a finite strain, deformable, solid element, the modifications required to extend such element into a beam are few. As explained in Sect. 2, only new interpolation functions and the global-to-local map (7) need to be programmed, and suitable quadrature rules should be selected.

In contrast, the implementation of a geometrically exact beam in any of the existing formulations is fairly involved due mainly to the treatment of the rotational degrees of freedom. This difficulty directly translates to the linearization of the formulation and the construction of time integration algorithms. This last aspect is treated below with more details. 
The difficulty in the implementation of GE beams does not refer only to the computation of the internal forces and tangent stiffness. The most crucial point is the update equations, in both quasi-static and transient problems, of the nodal rotational degrees of freedom and their rates. The required updates are fundamentally different to those commonly programmed in structural, finite element, and multibody codes. Including them in an existing code would require important modifications of the program at the global level.

\subsection{Numerical issues}

The original formulations of the ANC and GE beams suffered numerical problems that we only identified when they became popular and their use widespread. Modifications have been proposed which fix those issues to a great extent.

The most important numerical issue of the ANC formulation is that it has been shown to produce results converging to the wrong solution when Poisson's ratio is nonzero

In a nutshell, the kinematics of the continuum based ANC beams artificially couples shear and bending deformation and it is not rich enough to account for the section warping in torsion. This issue has been studied and solutions were found either by using high order interpolation functions, by using enhanced assumed strain formulations, or by leaving the continuum framework in favor of true beam theory. The first solutions require additional computational cost per element, and the latter loses many of the advantages that make continuum based ANC beams so appealing (simplicity, three-dimensional constitutive laws, etc. and so forth).

The geometrically exact beams formulation can also show locking in the thin limit if full integration is employed for the internal forces and the stiffness matrix. It is well known that a simple and cost-effective solution for such a problem is the use of selective reduced integration This well-known problem of Timoshenko-based finite elements, and its simple solution were reported in the first implementation of the method. A more subtle issue, which was not reported until is that many numerical implementations of GE beams spoil the objectivity of the exact equations of nonlinear beams. The reason can be traced back once again to the interpolation of the rotational degrees of freedom. More recent numerical implementations have been able to solve this problem by resorting to better interpolation strategies for such degrees of freedom.

\subsection{Interfacing with general material models}

In order to calculate nonlinear beams of arbitrary material types, it is necessary that the beam formulation allows a simple interface with general types of three-dimensional constitutive models. These can include hyperelastic materials, viscoelastic, elastoplastic, materials with damage, etc. Being in essence a continuum element, the $\mathrm{ANC}$ beams incorporate without any modification any three-dimensional constitutive model (see (10)).

In contrast, the GE formulation is based on section response laws. In this type of rule, the constitutive model is preintegrated across the beam section, a task that can only be done for very simple linear models. See, for example, the one shown in (15). For general materials, one can resort to iterative strategies such as the one advocated which is general but computationally expensive.

\subsection{Interface with structural models}

When engineers design structures with beams, they make constant use of stress resultants and section deformation measures ignoring, unless completely necessary, the details of the 
stress and strain distributions over the body. For the same reasons, external actions on the beam are applied in the form of point loads and concentrated moments or at most as distributed loads and moments per unit length. Body force fields or surface tractions of the type applied over continuum bodies are not employed at all.

If a beam finite element is to be used for structural analysis purposes, it is required that it can report section resultants and deformations, and that forces and moments can be applied in the manner described above. The GE beam of Sect. 3, being based on a beam theory, naturally fits in an structural analysis framework, and its interface with the user should be similar to any other (linear or nonlinear) beam element.

On the other hand, the ANC beam of Sect. 2, which is essentially a finite element for three-dimensional slender solids, does not make use of resultants or section strain measures. The former might be postprocessed from the stress field, if desired, but there does not seem to be a clear definition of all the classical section deformation measures for general ANC beams. Even more troublesome is the application of concentrated moments or imposing rotation angles at specific points of a structure. Since there are no degrees of freedom directly related with the rotations, the application of a simple bending moment can be very difficult and, for practical purposes, can be considered as not possible for these elements. See (13) for an illustration of this difficulty and for an explicit calculation of the value of the generalized forces corresponding to an external bending moment in a simple case.

\subsection{Dynamic problems}

Dynamic problems have specific issues which do not appear in quasi-static simulations. The first one, already mentioned in Sect. 3 concerns integration schemes. Again, due to its simple structure, ANC beams employ standard integration schemes without any special modification. This applies to linear multistep or Runge-Kutta methods, either implicit or explicit. In contrast, GE beam formulations require special time stepping methods for the numerical integration of the rotational degrees of freedom. This makes more difficult to incorporate such models into existing finite element or multibody codes.

Explicit integrations for mechanical problems require that the mass matrix be diagonal and constant. The first condition can be achieved in any case by lumping techniques, although a diagonal mass matrix does not lead to exact modeling of the section inertia. The second condition, while trivially satisfied by the ANC beams is violated by the GE finite element models. In them, the rotatory inertia is configuration dependent and the mass matrix needs to be recomputed in each time step. Recent results published indicate that a constant mass matrix could be developed for a GE formulation if some simplifications are accepted.

\subsection{Computational cost}

Computational cost should always be measured against accuracy. At this point, without any further information regarding the accuracy of either formulation, we can only establish some simple comparison that might give a rough idea of the computational cost per element and per node.

The simplest ANC beam has 24 degrees of freedom per element vs. 12 degrees of freedom for the two-noded GE beam. Moreover, in order to correctly integrate the element vectors and matrices of the ANC beam, a total of 16 integration points are required. For the GE element, only an average of 1.5 quadrature points are required ( 1 for the stiffness terms, 2 for the mass terms). This count allows to roughly estimate the cost of one ANC element to be about 10 times higher than the cost of one GE element. 
At the global level, for the same number of nodes in a mesh, the number of unknowns in roughly twice as large in the case of $\mathrm{ANC}$ beams, which amounts approximately to 4 to 8 times more $\mathrm{CPU}$ time just to solve the linearized equilibrium equations.

It must be mentioned that there exist $\mathrm{ANC}$ elements based on true beam theories that are much more efficient that the full "continuum-based" discussed above. As explained in Sect. 2, we have focused on the latter type, where all the advantages of complex material modeling can be readily implemented.

\section{Quantitative comparison}

In this section, we compare the results obtained with the two types of beam formulations when applied to benchmarks. All the examples are quasistatic to avoid errors due to the time integration, and to concentrate on the error that originate from the beam theory employed and the discretization. Of course, all these errors arise in dynamic simulations as well.

\subsection{Cantilever bent}

The first example was proposed

and serves to assess the accuracy and robustness of nonlinear rod implementations in situations with axial forces, bending, and torsion. It has been often used as a benchmark for accuracy in nonlinear finite elements.

The beam considered is clamped at one of its ends and a vertical point load is applied to its free end. The beam is depicted in Fig. 3, and its cross section is a square of unit side. The material is nonlinear elastic with Young's modulus $E=10^{7}$ and Poisson's ratio $v=0$. For the ANC beam, the material model is assumed to be Neohookean.

a mesh consisting of eight two-noded beams was considered. The same mesh is considered herein for the two formulations compared. Since the ANC beam has twice as many degrees of freedom per element as the GE one, a mesh with only four ANC elements is also taken into consideration. Also, a reference solution is presented obtained with a fine mesh of $50 \mathrm{GE}$ elements.

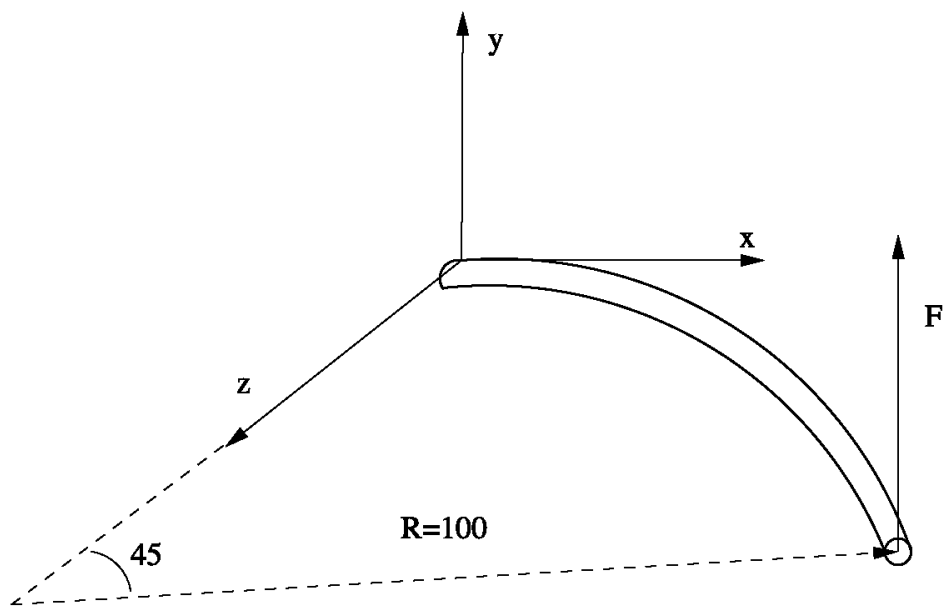

Fig. 3 Example 1. Geometry of the undeformed curved beam 
Table 1 Example 1. Initially curved beam. Cartesian components of the tip position under end forces of $F=300,450,600$. The initial coordinates of the tip are $(70.71,0,29.29)$

\begin{tabular}{|c|c|c|c|c|c|c|c|c|c|}
\hline & \multicolumn{3}{|c|}{$F=300$} & \multicolumn{3}{|c|}{$F=450$} & \multicolumn{3}{|c|}{$F=600$} \\
\hline & $r_{x}$ & $r_{y}$ & $r_{z}$ & $r_{x}$ & $r_{y}$ & $r_{z}$ & $r_{x}$ & $r_{y}$ & $r_{z}$ \\
\hline Bathe and Belourchi & 59.2 & 39.5 & 22.5 & - & - & - & 47.2 & 53.4 & 15.9 \\
\hline GE (8 elements) & 58.61 & 40.35 & 22.21 & 52.05 & 48.59 & 18.49 & 46.98 & 53.50 & 15.69 \\
\hline ANC (4 elements) & 64.78 & 28.68 & 25.66 & 62.14 & 33.94 & 24.04 & 59.95 & 37.55 & 22.71 \\
\hline ANC (8 elements) & 59.98 & 38.18 & 22.88 & 54.45 & 45.71 & 19.64 & 50.16 & 50.26 & 17.16 \\
\hline Reference solution & 58.54 & 40.48 & 22.12 & 51.97 & 48.70 & 18.37 & 46.89 & 53.60 & 15.56 \\
\hline
\end{tabular}

Table 2 Example 1. Euclidean norms of the errors in the tip displacement (compared with reference solution)

\begin{tabular}{lccc}
\hline & $F=300$ & $F=450$ & $F=600$ \\
\hline GE (8 elements) & 0.164 & 0.179 & 0.185 \\
ANC (4 elements) & 13.81 & 18.80 & 21.89 \\
ANC (8 elements) & 2.813 & 4.089 & 4.940 \\
\hline
\end{tabular}

Tables 1 and 2 summarize the results obtained. For each of the formulations and meshes considered, the position of the tip of the beam is indicated when the point load takes values $F=300,450$, and 600 . The results show that for this particular example, the GE formulation provides more accurate solutions. This is even more clear when we compare the two solutions obtained with the same number of degrees of freedom-the one with $8 \mathrm{GE}$ elements and the one with 4 ANC elements.

\subsection{Beam with slope discontinuity}

The numerical treatment of ANC beams with nonsmooth centroid curves requires special attention, and was studied In the next example, we consider a structure consisting of three straight beams of unit length connected at right angles. Figure 4 depicts the geometry of the model, the cross section, and the applied loading. The Young's modulus and Poisson's ratio of the material are $E=10^{6}$ and $\nu=0$, respectively.

The deformation of the beam under the application of forces at the tip includes large deformations and displacements. Figure 5 shows the evolution of the tip displacement as a function of the modulus $F$ of the applied loads for a mesh consisting of 12 identical ANC elements. It can be verified that the displacements for the maximum value $F=5$ are of the same order of magnitude as the length of the beams.

We proceed to study the behavior of the error in the tip displacement as a function of the mesh size. For that, we consider the solution of the beam using meshes with $3,6,12$, 24 , and 48 elements. To measure the error, we calculate a reference solution obtained with a fine mesh of $120 \mathrm{GE}$ elements. In Figs. 6 and 7, logarithmic plots of the error evolution is depicted as a function of the number of elements and the number of degrees of freedom in each computation.

As in the previous example, the computations show that the GE formulation provides more accurate solutions for the same number of elements. The difference is even more dramatic if we consider the accuracy for the same number of degrees of freedom, which is, for the example under study, of two orders of magnitude. 
Fig. 4 Example 2. Geometry of the structure, cross section, and loading
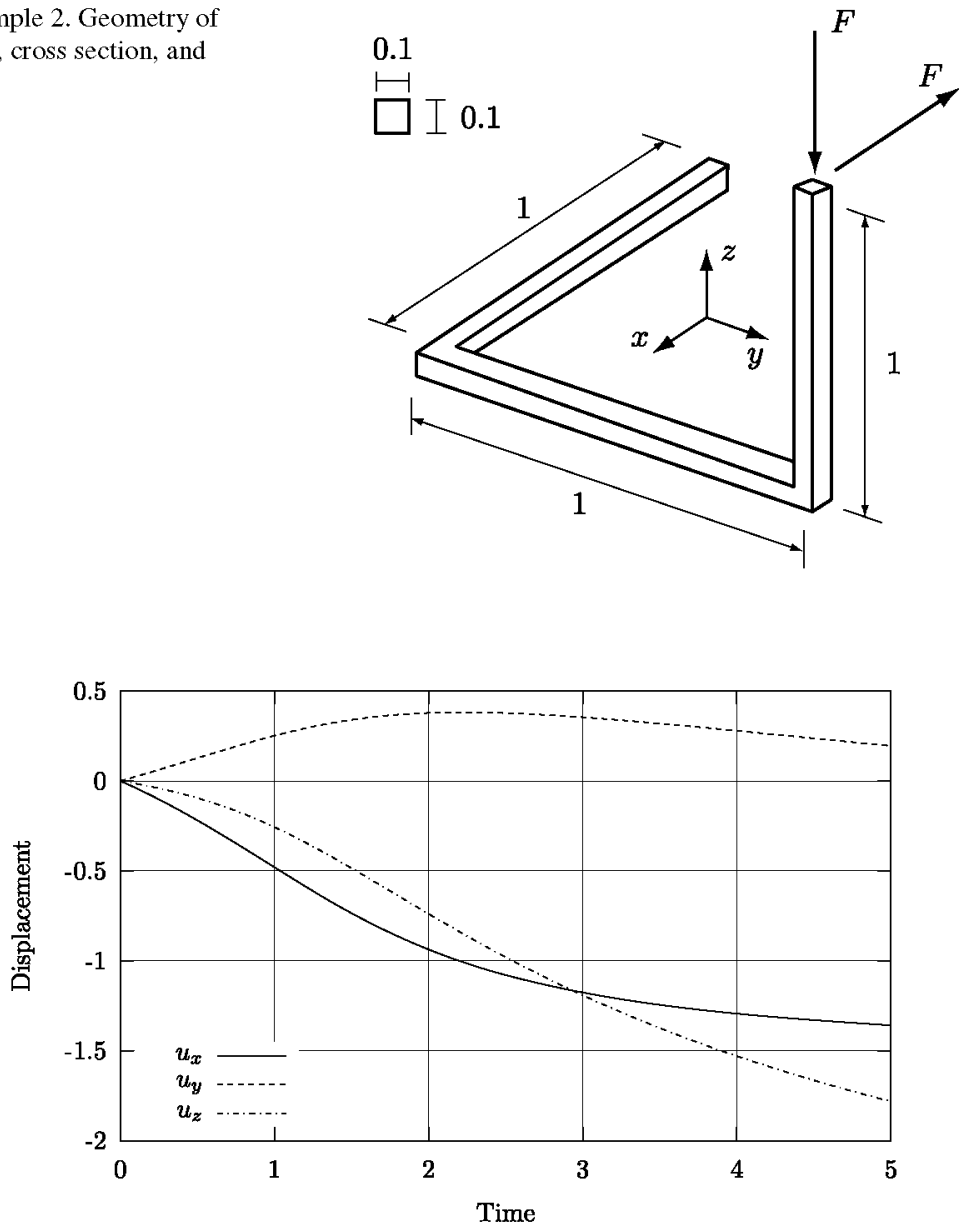

Fig. 5 Example 2. Tip displacements as functions of the applied forces. Mesh with 12 ANC elements

\subsection{Poisson's locking}

It has been reported, that when the Poisson's ratio is not zero, the ANC formulation might converge to a solution different from the exact one, even for very simple problems such a straight cantilever beam under concentrated loading. To show that the GE formulation does not suffer such drawback, Figs. 8 and 9 show the relative tip displacement errors in a cantilever beam of unit length, with square cross section of side 0.1 , and Young's modulus $E=10^{6}$, under a vertical point load of unit value. Figure 8 shows the results for Poisson's ratio $v=0$ and Fig. 9 for $v=0.3$, using as reference the analytical solution. The curves clearly show that for nonvanishing Poisson's ratio, the ANC beam does not converge to the exact solution, whereas the GE formulation does. For $v=0$, both methods converge. Consistent with the results of the previous examples, in this second case, the GE formulation provides more accurate results for the same number of degrees of freedom. 


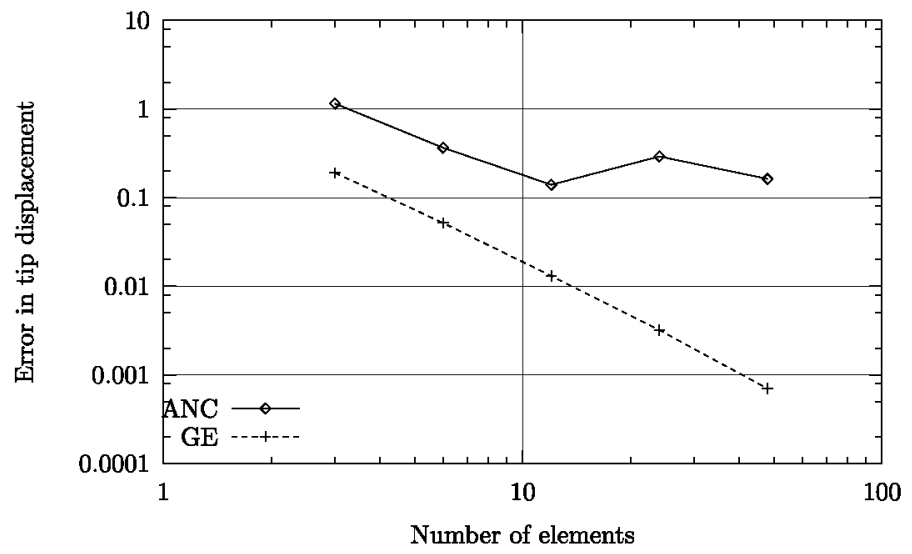

Fig. 6 Example 2. Errors in tip displacements as a function of the number of elements

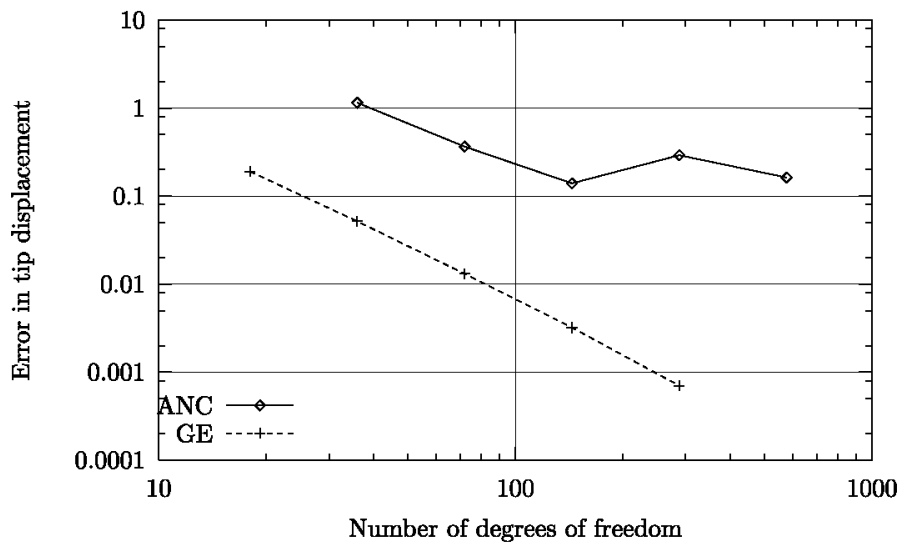

Fig. 7 Example 2. Errors in tip displacements as a function of the number of degrees of freedom

\subsection{Locking in the thin limit}

In the next example, we explore numerically the accuracy of the beam models as the thickto-span ratio is very changes. A cantilever beam has a vertical point load of unit modulus applied to its free end. The material is elastic with Young's modulus $E=10^{6}$ and Poisson's ratio $v=0.3$. We consider a series of linear problems that consist of four beams with cross sections of sides $h=0.1,0.05,0.02$, and 0.01 . We compare the analytical solutions to these problems with the solutions obtained with the ANC and the GE formulation, the latter with full and reduced integration. In all cases, a mesh consisting of 10 equal elements is employed.

Figure 10 shows the result obtained, clearly identifying the locking of the GE formulation when full integration is employed: in this case, as the thickness-to-span ratio diminishes, the relative errors are of the order of 1 . In the other two implementation, no locking of this type appears. The GE implementation with reduced integration shows more accuracy for every value of the comparison parameter, but the accuracy of the ANC beam does not get worse when the ratio thickness-to-span goes to zero. We would like to stress that Fig. 10 is not 


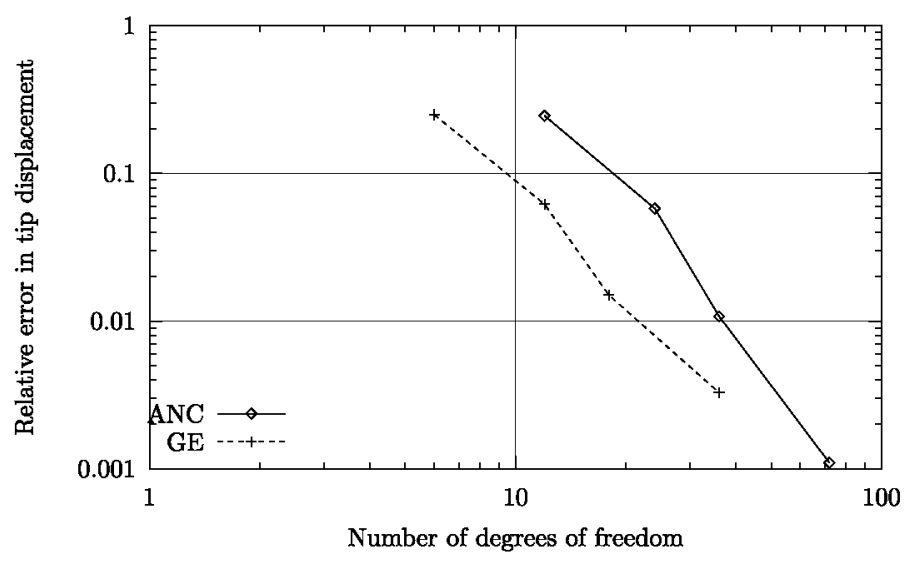

Fig. 8 Example 3. Relative tip displacements error in cantilever beam for $v=0$

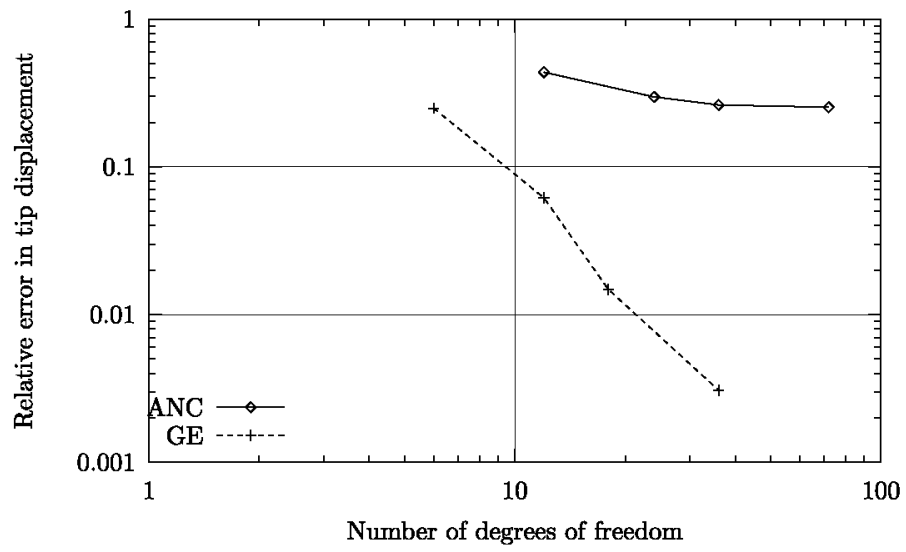

Fig. 9 Example 3. Relative tip displacements error in cantilever beam for $v=0.3$

a convergence plot, and the relative error need not approach zero as the thickness-to-span ratio does.

\section{Summary and conclusions}

In this article, we have compared the absolute nodal coordinate and geometrically exact beam formulations, two of the most popular approaches for discretizing nonlinear beams within the contexts of finite elements and multibody systems. While these two families of methods have evolved in the last years, and each of them exists in several different "flavors," we have focused on the original implementations

Sections 4 and 5 discuss qualitative and quantitative issues of the two formulations, highlighting the pros and cons of each of them. We have tried to cover a wide range of aspects, including implementation effort, performance, robustness, interfacing with material models and other structural members, and accuracy. 


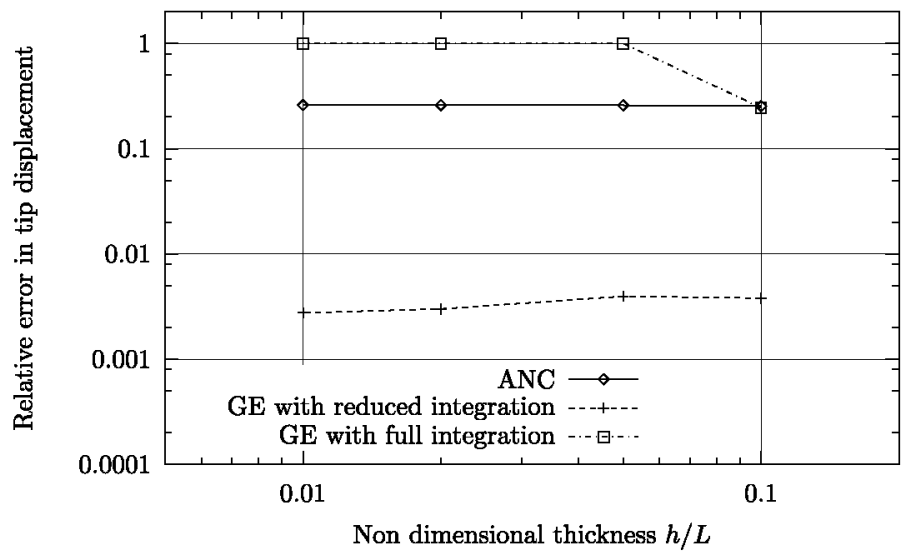

Fig. 10 Example 3. Relative tip displacements error in cantilever beam

A first observation is that the ANC is the only formulation possible for those codes that do not work with rotation variables. In addition to this restriction, ANC beams might be considered when a general purpose element is required which is simple to program and to include in a exiting finite element or multibody code. The generality of its formulation allows to work with complex material models (elastic or inelastic) without any additional difficulty. The dynamic equations of the element are also very simple and their time integration standard.

Regarding the elements based on geometrically exact beam theory, they are the formulation of choice when performance and accuracy are crucial. Despite their complexity, both theoretical and numerical, our results strongly indicate that for the same computational cost, these types of elements provide fairly more accurate results than the ANC elements. The CPU cost involved in the computations of one ANC element is also significantly higher than in the GE case.

In engineering analysis, structural models need to sustain applied concentrated moments and imposed rotations. Any type of GE element can be used for such a purpose, whereas the ANC elements lack these features, making the former more suitable for engineering structural analysis.

As a final conclusion, we can not claim the superiority of one approach over the other. The recommendations given above and the results of Sects. 4 and 5 can provide guidelines for the appropriate choice of formulation, which in most cases will depend on the foreseen applications and the restrictions imposed by the existing code where this type of element needs to be implemented.

Acknowledgements The financial support from the Spanish Ministry of Science and Education through grant DPI2006-14104 is gratefully acknowledged.

\section{References}

Antman, S.S.: The Theory of Rods. Handbuch der Physik, vol. VIa/2. Springer, Berlin (1972) (Flügge, S., Truesdell, C. (eds.))

Argyris, J.H.: An excursion into large rotations. Comput. Methods Appl. Mech. Eng. 32, 85-155 (1982)

Avello, A., García de Jalón, J.: Dynamics of flexible multibody systems using Cartesian co-ordinates and large displacement theory. Int. J. Numer. Methods Eng. 32, 1543-1563 (1991) 
Bathe, K.J.: Finite Element Procedures. Prentice Hall, Englewood Cliffs (1996)

Bathe, K.J., Belourchi, S.: Large displacement analysis of three-dimensional beam structures. Int. J. Numer. Methods Eng. 14, 961-986 (1979)

Bauchau, O.A., Theron, N.J.: Energy decaying scheme for non-linear beam models. Comput. Methods Appl. Mech. Eng. 134(1-2), 37-56 (1996)

Betsch, P., Menzel, A., Stein, E.: On the parametrization of finite rotations in computational mechanics. A clarification of concepts with application to smooth shells. Comput. Methods Appl. Mech. Eng. 155, 273-305 (1998)

Betsch, P., Steinmann, P.: Frame-indifferent beam element based upon the geometrically exact beam theory. Int. J. Numer. Methods Eng. 54, 1775-1788 (2002)

Betsch, P., Steinmann, P.: Constrained dynamics of geometrically exact beams. Comput. Mech. 31, 49 59 (2003)

Bonet, J., Wood, R.D.: Nonlinear Continuum Mechanics for Finite Element Analysis. Cambridge University Press, Cambridge (1997)

Botasso, C.L., Borri, M.: Integrating finite rotations. Comput. Methods Appl. Mech. Eng. 164, 307-331 (1998)

Cardona, A., Geradin, M.: A beam finite element nonlinear theory with finite rotations. Int. J. Numer. Methods Eng. 26, 2403-2434 (1988)

Crisfield, M.A., Galvanetto, G., Jelenić, U.: Dynamics of 3D co-rotational beams. Comput. Mech. 20(6), 507-519 (1997)

Crisfield, M.A., Jelenić, U.: Objectivity of strain measures in geometrically exact 3D beam theory and its finite element implementation. Proc. R. Soc. Lond. Ser. A 455, 1125-1147 (1999)

Crisfield, M.A.: Non-linear Finite Element Analysis of Solids and Structures. Advanced Topics, vol. 2. Wiley, New York (1997)

Ericksen, J.L., Truesdell, C.: Exact theory of stress and strain in rods and shells. Arch. Ration. Mech. Anal. 1, 195-323 (1958)

Gerstmayr, J., Matikainen, M.K.: Analysis of stress and strain in the absolute nodal coordinate formulation. Mech. Des. Struct. Mach. 34(4), 409-430 (2006)

Gerstmayr, J., Shabana, A.A.: Efficient integration of the elastic forces and thin three-dimensional beam elements in the absolute nodal coordinate formulation. In: García Orden, J.C., Goicolea, J.M., Cuadrado, J. (eds.) Multibody Dynamics 2005. ECCOMAS Thematic Conference, Madrid, Spain, 2124 June 2005

Gerstmayr, J., Shabana, A.A.: Analysis of thin beams and cables using the absolute nodal coordinate formulation. Nonlinear Dyn. 45(1-2), 109-130 (2006)

Green, A.E., Laws, N.: A general theory of rods. Proc. R. Soc. Lond. Ser. A 293, 145-155 (1966)

Green, A.E., Naghdi, P.M., Wenner, M.L.: On the theory of rods I derivations from the three dimensional equations. Proc. R. Soc. Lond. Ser. A 337, 451-483 (1974)

Green, A.E., Naghdi, P.M., Wenner, M.L.: On the theory of rods II developments by direct approach. Proc. R. Soc. Lond. Ser. A 337, 485-507 (1974)

Hughes, T.J.R.: The Finite Element Method. Prentice-Hall, Englewood Cliffs (1987)

Ibrahimbegovic, A.: On finite element implementations of geometrically nonlinear Reissner's beam theory: three-dimensional curved beam elements. Comput. Methods Appl. Mech. Eng. 122, 11-26 (1995)

Ibrahimbegovic, A., Al Mikdad, M.: Finite rotations in dynamics of beams and implicit time-stepping algorithms. Int. J. Numer. Methods Eng. 41(5), 781-814 (1998)

Jelenić, G., Crisfield, M.A.: Interpolation of rotational variables in nonlinear dynamics of 3D beams. Int. J. Numer. Methods Eng. 43, 1193-1222 (1998)

Jelenić, G., Crisfield, M.A.: Geometrically exact 3D beam theory: implementation of a strain-invariant finite element for statics and dynamics. Comput. Methods Appl. Mech. Eng. 171, 141-171 (1999)

Klinkel, S., Govindjee, S.: Using finite strain 3D-material models in beam and shell elements. Eng. Comput. 19(8), 902-921 (2002)

Lens, E.V., Cardona, A.: A nonlinear beam element formulation in the framework of an energy preserving time integration scheme for constrained multibody systems dynamics. Comput. Struct. 86, 47-63 (2008) Maqueda, L.G., Bauchau, O.A., Shabana, A.A.: Effect of the centrifugal forces on the finite element eigenvalue solution of a rotating blade: a comparative study. Multibody Syst. Dyn. (2007). doi: $10.1007 /$ s11044-007-9070-6

Reissner, E.: A one-dimensional finite strain beam theory: the plane problem. J. Appl. Math. Phys. (ZAMP) 23, 795-804 (1972)

Romero, I.: The interpolation of rotations and its application to finite element models of geometrically exact rods. Comput. Mech. 34(2), 121-133 (2004)

Romero, I., Armero, F.: An objective finite element approximation of the kinematics of geometrically exact rods and its use in the formulation of an energy-momentum conserving scheme in dynamics. Int. J. Numer. Methods Eng. 54(12), 1683-1716 (2002) 
Sansour, C., Wagner, W.: Multiplicative updating of the rotation tensor in the finite element analysis of rods and shells-a path independent approach. Comput. Mech. 31, 153-162 (2003)

Schwab, A.L., Meijaard, J.P.: Comparison of three-dimensional flexible beam elements for dynamic analysis: finite element method and absolute nodal coordinate formulation. In: IDETC/CIE 2005 ASME International Design Engineering Technical Conferences, Long Beach, CA, Sept. 2005

Shabana, A.A., Mikkola, A.M.: Use of the finite element absolute nodal coordinate formulation in modeling slope discontinuity. Trans. Am. Soc. Mech. Eng. J. Mech. Des. 125, 342-350 (2003)

Shabana, A.A., Yakoub, R.Y.: Three dimensional absolute nodal coordinate formulation for beam elements: theory. ASME J. Mech. Des. 123(4), 606-613 (2001)

Simo, J.C.: A finite strain beam formulation. Part I. The three-dimensional dynamic problem. Comput Methods Appl. Mech. Eng. 49, 55-70 (1985)

Simo, J.C., Tarnow, N., Doblaré, M.: Non-linear dynamics of three-dimensional rods: exact energy and momentum conserving algorithms. Int. J. Numer. Methods Eng. 38(9), 1431-1473 (1995)

Simo, J.C., Vu-Quoc, L.: On the dynamics of flexible beams under large overall motions-the plane case II. Trans. Am. Soc. Mech. Eng. J. Appl. Mech. 53(4), 855-63 (1986)

Simo, J.C., Vu-Quoc, L.: A three-dimensional finite-strain rod model Part II: Computational aspects. Comput. Methods Appl. Mech. Eng. 58, 79-116 (1986)

Simo, J.C., Vu-Quoc, L.: On the dynamics in space of rods undergoing large motions-a geometrically exact approach. Comput. Methods Appl. Mech. Eng. 66(2), 125-161 (1988)

Smoleński, W.M.: Statically and kinematically exact nonlinear theory of rods and its numerical verification. Comput. Methods Appl. Mech. Eng. 178, 89-1 13 (1999)

Sopanen, J.T., Mikkola, A.M.: Description of elastic forces in absolute nodal coordinate formulation. Nonlinear Dyn. 34, 53-74 (2003)

Yacoub, R.Y., Shabana, A.A.: Three dimensional absolute nodal coordinate formulation for beam elements: implementation and applications. Am. Soc. Mech. Eng. J. Mech. Eng. 123(4), 614-621 (2001)

Zienkiewicz, O.C., Taylor, R.L.: The Finite Element Method, 4th edn. McGraw-Hill, New York (1991) 